\title{
An Efficient Estimator for Target Localization in a Multistation Redundancy System without Matrix Inversion
}

\author{
Xiwen Zhang $\mathbb{D}^{\mathbb{D}}$, Jiyan Huang $\mathbb{D}^{\mathrm{D}}$, Yiyuan Wang ${ }^{\mathbb{D}}$, and Yineng Zhou \\ School of Information and Communication Engineering, University of Electronic Science and Technology of China (UESTC), \\ Chengdu 611731, China \\ Correspondence should be addressed to Jiyan Huang; huangjiyan@uestc.edu.cn
}

Received 22 March 2018; Revised 30 May 2018; Accepted 6 June 2018; Published 16 July 2018

Academic Editor: Raymond Swartz

Copyright (C) 2018 Xiwen Zhang et al. This is an open access article distributed under the Creative Commons Attribution License, which permits unrestricted use, distribution, and reproduction in any medium, provided the original work is properly cited.

\begin{abstract}
This paper focuses on target localization problem in a multistation redundancy system which finds broad applications in sonar, radar, and location-based service. Previous solutions can only be applied to the minimum system, such as the TOA method with three sensors and the AOA method with two sensors or need matrix inversion. To solve this problem, we propose a simple closed-form solution for a multistation redundancy localization system by using the estimation variance as the weighting coefficient to compute an average of each group's localization result. The proposed method, with simple algebraic solution, requires no matrix inversion and can be used for low-cost hardware devices. We derive the method in TOA solution and AOA solution, respectively. The proposed method can also be extended to other locating technologies. Numerical examples are provided to illustrate the performance of the proposed method in root-mean-square error. The positioning accuracy of the proposed method is close to Cramér-Rao low bound both in TOA solution and AOA solution.
\end{abstract}

\section{Introduction}

Target localization has a wide range of applications in sonar, radar, and location-based service [1]. It is often of concern for many scholars to enhance the localization accuracy and make the localization algorithm widely applicable to all kinds of situations [2]. A large variety of range-based localization methods have been proposed with respect to a set of sensors with known positions [3, 4]. Several range-based geolocation techniques have been used to estimate the target position including the time-of-arrival (TOA) [5-7], the time-difference-of-arrival (TDOA) [8, 9], the angle-of-arrival (AOA) [10-12], the received signal strength (RSS) [13-16] based techniques, or hybrid location techniques $[17,18]$. Among these location techniques, TOA and AOA have attracted much attention because of their high positioning accuracy $[16,18]$. This paper focuses on TOA and AOA location techniques.

There are algebraic method and matrix method for the multistation localization system [5]. Algebraic methods mainly include the TOA method [19] which uses the circles produced by range measurements at other sensors to find the position of the transmitter at the intersection of circles in two dimensions or spheres in three dimensions and the AOA method [10] which calculates the target position from the point of intersection of bearing lines. It should be noted that algebraic methods are designed for the minimum location system, such as the TOA method with three anchor sensors, and the AOA method with two anchor sensors. The main advantage of algebraic methods is that they can provide a simple algebraic solution without the computation of matrix inversion which makes those methods can be applied in a low-cost location system especially for wireless sensor networks (WSNs) [5, 20]. Since the algebraic methods are based on local information of a minimum system, the direct extension for those methods to a multistation redundancy system may not obtain optimal performance since those solutions cannot make use of extra measurements to improve position accuracy [8].

To improve the localization accuracy, matrix methods $[5,6,8,11,21-25]$ based on global information of measurements from all sensors were developed for a multistation 
redundancy system. The closed-form solution which requires solving a set of linear equations including all the measurements was given by Friedlander [21]. In order to obtain optimal estimation, Foy proposed an iterative localization method [26] based on Taylor series expansion. It starts with an initial guess and improves the estimate at each step by determining the local linear least-squares (LS) solution. Moreover, a proportion of solutions concentrates on transforming the localization problem into a manageable form. The LS method [6] based on TOA measurements is provided by Caffery. It uses the least square procedure to solve geometric equations which represent the lines of positions. Chan's method [8], using a two-step weighted LS approach, provides higher accuracy which is close to CRLB when the number of sensors is greater than 3 . Since the above matrix methods are based on global information and consider the relationships among all of measurements, they have better performance than the algebraic method and may achieve the CRLB. Unfortunately, the high computational burden caused by matrix inversion makes it hard for matrix methods to be applied for a low-cost location system such as WSNs with several Mhz oscillators [20, 27].

It is necessary to develop a location scheme with high positioning accuracy for a low-cost location system. In this paper, a novel and efficient estimator is designed for a low-cost location system. The proposed method first divides all of anchor sensors into several groups. Each group is a minimum location system and can provide a position estimate using an algebraic method. Subsequently, theoretical variances of position estimates for every group are derived and are used as weights to obtain the final solution. The proposed method utilizes groups to avoid matrix inversion and uses theoretical variances as weights to improve the system performance. In this paper, the proposed method is applied in TOA and AOA location techniques. Moreover, the theoretical variance of the proposed method is also derived for performance analysis. Simulation results verify the proposed method. Compared with current studies, the proposed method not only provides a simple algebraic solution without matrix inversion but also has the similar performance with the matrix method.

To illustrate, the paper is organized as follows. Section 2 formulates the system model of the localization problem in a multistation redundancy system using TOA measurements and AOA measurements in a 2-D plane, respectively. The previous method is described and analyzed. Section 3 presents the proposed method in TOA solution and AOA solution, respectively. The performance of the proposed method is analyzed by deriving the theoretical estimate variance in Section 4. Simulation results are presented in Section 5 to illustrate the localization accuracy compared with CRLB and other methods. Section 6 concludes this paper.

\section{Problem Formulation}

The target localization problem in a multistation redundancy system which contains $N$ anchor sensors is depicted in Figure 1. For notational convenience and ease of illustration,

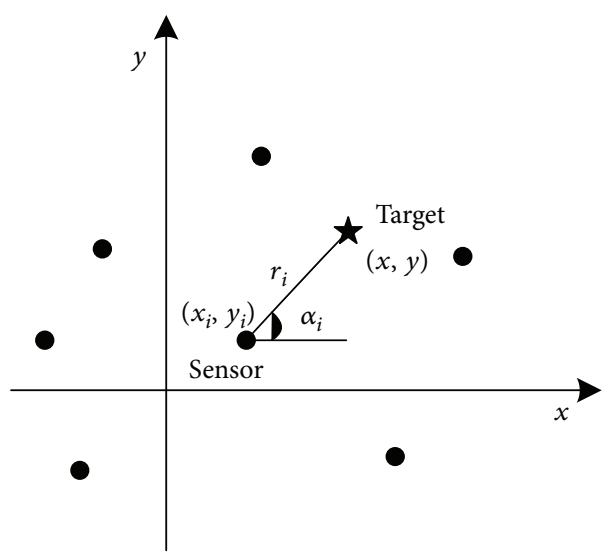

FIgURE 1: Localization in a multistation redundancy system.

we model the problem in a 2-D plane. Extension to three dimensions is straightforward. Assume that $N$ anchor sensors are located at known positions $\left(x_{i}, y_{i}\right), i=1,2, \ldots$, $N$ and the unknown target position is $(x, y)$. The objective of the target localization is to identify the target location from the known observers' positions and the measurements, such as TOAs and AOAs.

Assume that TOA and AOA measurements have already been measured as shown in Figure 1. The measured distance between the target and ith sensor is $r_{i}$ and the estimation of azimuth for the target is $\alpha_{i}$. Then, we can use these measurements to establish TOA equations [28]

$$
r_{i}^{2}=\left(x_{i}-x\right)^{2}+\left(y_{i}-y\right)^{2}, \quad i=1,2, \ldots, N,
$$

and $\mathrm{AOA}$ equations [10]

$$
y-y_{i}=\tan \alpha_{i}\left(x-x_{i}\right), \quad i=1,2, \ldots, N .
$$

Let $k_{i}=x_{i}^{2}+y_{i}^{2}$, and $r=x^{2}+y^{2}$. So that (1) can be written as

$$
r_{i}^{2}-k_{i}=-2 x_{i} x-2 y_{i} y+r, \quad i=1,2, \ldots, N,
$$

which is a set of linear equations with unknowns $x, y$, and $r$. From (2) we can obtain

$$
\sin \alpha_{i} x-\cos \alpha_{i} y=\sin \alpha_{i} x_{i}-\cos \alpha_{i} y_{i}, \quad i=1,2, \ldots, N
$$

Since TOA and AOA measurements have error, which consists of the true measurements corrupted by additive independent zero-mean Gaussian noise. So with TOA and AOA noise, the error vector $[11,28]$ derived from (3) and (4) is

$$
\varphi=\mathbf{h}-\mathbf{G z}
$$

where 


$$
\begin{aligned}
\mathbf{h} & =\left[\begin{array}{c}
r_{1}^{2}-k_{1} \\
r_{2}^{2}-k_{2} \\
\vdots \\
r_{N}^{2}-k_{N}
\end{array}\right], \\
\mathbf{G} & =\left[\begin{array}{ccc}
-2 x_{1} & -2 y_{1} & 1 \\
-2 x_{2} & -2 y_{2} & 1 \\
\ldots & \ldots & \ldots \\
-2 x_{N} & -2 y_{N} & 1
\end{array}\right], \\
\mathbf{z} & =\left[\begin{array}{c}
x \\
y \\
r
\end{array}\right],
\end{aligned}
$$

in TOA case,

$$
\begin{aligned}
& \mathbf{h}=\left[\begin{array}{c}
\sin \alpha_{1} x_{1}-\cos \alpha_{1} y_{1} \\
\sin \alpha_{2} x_{2}-\cos \alpha_{2} y_{2} \\
\vdots \\
\sin \alpha_{N} x_{N}-\cos \alpha_{N} y_{N}
\end{array}\right], \\
& \mathbf{G}=\left[\begin{array}{cc}
\sin \alpha_{1} & -\cos \alpha_{1} \\
\sin \alpha_{2} & -\cos \alpha_{2} \\
\ldots & \ldots \\
\sin \alpha_{N} & -\cos \alpha_{N}
\end{array}\right], \\
& \mathbf{z}=\left[\begin{array}{c}
x \\
y
\end{array}\right],
\end{aligned}
$$

in AOA case.

Most of the previous methods [5, 6, 8, 11, 23, 25] are based on the least square procedure. Thus, the source position is computed by

$$
\mathbf{Z}=\left(\mathbf{G}^{\mathrm{T}} \mathbf{G}\right)^{-\mathbf{1}} \mathbf{G}^{\mathrm{T}} \mathbf{h},
$$

or some improvements of it, such as the two-step weighted LS method (Chan's method) [8]. These methods require matrix inversion that leads to a high requirement for hardware devices. However, low-cost hardware devices, such as some embedded systems, may not support matrix inversion. Hence, it is difficult for these methods to be applied to a low-cost location system. A more widely adapted method needs to be developed to address this situation.

\section{The Proposed Method}

In order to enable low-cost hardware devices to achieve high-precision positioning, we propose a simple closedform solution for the multistation localization system.
Without matrix inversion, the proposed method provides an algebraic solution which can be effectively applied to low-cost hardware devices.

We develop the approach by using theoretical variances as weighting coefficients to compute an average of each group's localization result. As shown in Figure 2, first, the anchor sensors are divided into several groups and each group of sensors are used to estimate the target position, respectively. Then, theoretical variances of the position estimates in each group are calculated and regarded as weighted values. Finally, the weighted average of the estimates is obtained as final solution.

TOA and AOA measurements have already been measured, and the specific derivations are as follows.

3.1. TOA Solution. Assume that there are $N$ anchor sensors distributed arbitrarily in a 2-D plane. We divide these sensors into $M$ groups. Each group has 3 sensors, which are the minimum number of base stations required for the TOA location algorithm. Note that some sensors may be repeated in different groups. In the $i$ th group, the $m$ th observation station is at known position $\left(x_{i m}, y_{i m}\right)$ and the distance measured between the target and $m$ th sensor is $\widehat{d}_{i m}$, for $i=1,2, \ldots, M$.

In each group, the localization scenario consists of a source and three observation stations. Assume that the unknown source position is located at $(x, y)$. Then, the TOA measurement equations [19] in $i$-th group are given by

$$
d_{i m}=\sqrt{\left(x_{i m}-x\right)^{2}+\left(y_{i m}-y\right)^{2}}, \quad m=1,2,3,
$$

where $d_{i m}$ is the true distance between the target and $m$ th sensor.

Transform (9) with respect to the first receiver, we obtain

$$
\left(x_{i 2}-x_{i 1}\right) x+\left(y_{i 2}-y_{i 1}\right) y=\frac{1}{2}\left[\left(x_{i 2}^{2}+y_{i 2}^{2}\right)-\left(x_{i 1}^{2}+y_{i 1}^{2}\right)+d_{i 1}^{2}-d_{i 2}^{2}\right],
$$

$\left(x_{i 3}-x_{i 1}\right) x+\left(y_{i 3}-y_{i 1}\right) y=\frac{1}{2}\left[\left(x_{i 3}^{2}+y_{i 3}^{2}\right)-\left(x_{i 1}^{2}+y_{i 1}^{2}\right)+d_{i 1}^{2}-d_{i 3}^{2}\right]$.

Using (10) and (11), we obtain

$$
\begin{aligned}
& x=\frac{\left(y_{i 2}-y_{i 1}\right) c_{i 3}-\left(y_{i 3}-y_{i 1}\right) c_{i 2}}{\left(x_{i 3}-x_{i 1}\right)\left(y_{i 2}-y_{i 1}\right)-\left(x_{i 2}-x_{i 1}\right)\left(y_{i 3}-y_{i 1}\right)}, \\
& y=\frac{\left(x_{i 2}-x_{i 1}\right) c_{i 3}-\left(x_{i 3}-x_{i 1}\right) c_{i 2}}{\left(y_{i 3}-y_{i 1}\right)\left(x_{i 2}-x_{i 1}\right)-\left(y_{i 2}-y_{i 1}\right)\left(x_{i 3}-x_{i 1}\right)} .
\end{aligned}
$$

Since TOA measurements have errors, which consist of the true TOAs corrupted by additive independent zeromean Gaussian noise, we actually model distance estimates associated with receiver $m$ as

$$
\widehat{d}_{i m}=\widehat{d}_{i m}+n_{i m}, \quad m=1,2,3,
$$

where $n_{i m} \sim N\left(0, \sigma^{2}\right)$ and $m=1,2,3$. 


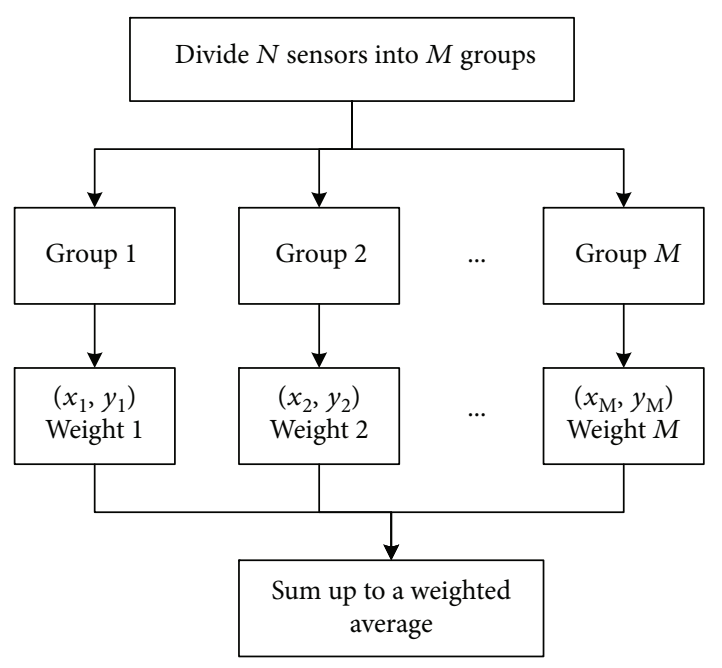

FIgURE 2: The process of the proposed method.

Let $\widehat{x}_{i}$ and $\hat{y}_{i}$ represent the position estimate coordinates in each group, where subscript $i$ denotes the $i$ th group. Then, the location estimate is

$$
\begin{aligned}
& \widehat{x}_{i}=\frac{\left(y_{i 2}-y_{i 1}\right) \widehat{c}_{i 3}-\left(y_{i 3}-y_{i 1}\right) \widehat{c}_{i 2}}{\left(x_{i 3}-x_{i 1}\right)\left(y_{i 2}-y_{i 1}\right)-\left(x_{i 2}-x_{i 1}\right)\left(y_{i 3}-y_{i 1}\right)}, \\
& \hat{y}_{i}=\frac{\left(x_{i 2}-x_{i 1}\right) \widehat{c}_{i 3}-\left(x_{i 3}-x_{i 1}\right) \widehat{c}_{i 2}}{\left(y_{i 3}-y_{i 1}\right)\left(x_{i 2}-x_{i 1}\right)-\left(y_{i 2}-y_{i 1}\right)\left(x_{i 3}-x_{i 1}\right)},
\end{aligned}
$$

where $\widehat{c}_{i 2}=1 / 2\left[\left(x_{i 2}^{2}+y_{i 2}^{2}\right)-\left(x_{i 1}^{2}+y_{i 1}^{2}\right)+\widehat{d}_{i 1}^{2}-\widehat{d}_{i 2}^{2}\right]$ and $\widehat{c}_{i 3}=$ $1 / 2\left[\left(x_{i 3}^{2}+y_{i 3}^{2}\right)-\left(x_{i 1}^{2}+y_{i 1}^{2}\right)+\widehat{d}_{i 1}^{2}-\widehat{d}_{i 3}^{2}\right]$.

With 3 sensors, the target position can be solved using (15) in each group. We compute theoretical variance by using the differential method [8]. From (12), the differential of $x_{i}$ is

$$
\Delta x_{i}=a_{i 3} \Delta c_{i 3}-a_{i 2} \Delta c_{i 2},
$$

where

$$
\begin{aligned}
& a_{i 3}=\frac{\left(y_{i 2}-y_{1 i}\right)}{\left(x_{i 3}-x_{i 1}\right)\left(y_{i 2}-y_{i 1}\right)-\left(x_{i 2}-x_{i 1}\right)\left(y_{i 3}-y_{i 1}\right)}, \\
& a_{i 2}=\frac{\left(y_{i 3}-y_{i 1}\right)}{\left(x_{i 3}-x_{i 1}\right)\left(y_{i 2}-y_{i 1}\right)-\left(x_{i 2}-x_{i 1}\right)\left(y_{i 3}-y_{i 1}\right)},
\end{aligned}
$$

$\Delta c_{i 2}=d_{i 1} \Delta d_{1 i}-d_{i 2} \Delta d_{i 2}$, and $\Delta c_{i 3}=d_{i 1} \Delta d_{i 1}-d_{i 3} \Delta d_{i 3}$. The differential of $y_{i}$ is

$$
\Delta y_{i}=b_{i 3} \Delta c_{i 3}-b_{i 2} \Delta c_{i 2},
$$

where

$$
\begin{aligned}
& b_{i 3}=\frac{\left(x_{i 2}-x_{i 1}\right)}{\left(y_{i 3}-y_{i 1}\right)\left(x_{i 2}-x_{i 1}\right)-\left(y_{i 2}-y_{i 1}\right)\left(x_{i 3}-x_{i 1}\right)}, \\
& b_{i 2}=\frac{\left(x_{i 3}-x_{i 1}\right)}{\left(y_{i 3}-y_{i 1}\right)\left(x_{i 2}-x_{i 1}\right)-\left(y_{i 2}-y_{i 1}\right)\left(x_{i 3}-x_{i 1}\right)} .
\end{aligned}
$$

For simplicity, we assume that the signal and noises are mutually independent, zero-mean stationary Gaussian random processes. If the distance estimation variance is $\sigma^{2}$, then the theoretical variance of $x_{i}$ is

$$
\begin{aligned}
\operatorname{var}\left(x_{i}\right) & =E\left[\Delta x_{i}^{2}\right]=a_{i 3}^{2} E\left[\Delta c_{i 3}^{2}\right]-2 a_{i 3} a_{i 2} E\left[\Delta c_{i 2} \Delta c_{i 3}\right]+a_{i 2}^{2}\left[\Delta c_{i 2}^{2}\right] \\
& =\left(a_{i 3}-a_{i 2}\right)^{2} d_{i 1}^{2} \sigma^{2}+a_{i 3}^{2} d_{i 3}^{2} \sigma^{2}+a_{i 2}^{2} d_{i 2}^{2} \sigma^{2} .
\end{aligned}
$$

The theoretical variance of $y_{i}$ is

$$
\begin{aligned}
\operatorname{var}\left(y_{i}\right) & =E\left[\Delta y_{i}^{2}\right]=b_{i 3}^{2} E\left[\Delta c_{i 3}^{2}\right]-2 b_{i 3} b_{i 2} E\left[\Delta c_{i 2} \Delta c_{i 3}\right]+b_{i 2}^{2}\left[\Delta c_{i 2}^{2}\right] \\
& =\left(b_{i 3}-b_{i 2}\right)^{2} d_{i 1}^{2} \sigma^{2}+b_{i 3}^{2} d_{i 3}^{2} \sigma^{2}+b_{i 2}^{2} d_{i 2}^{2} \sigma^{2}
\end{aligned}
$$

The variance weighted average TOA solution is given by

$$
\begin{aligned}
& \widehat{x}_{\text {wa_TOA }}=\frac{\sum_{i=1}^{M} \hat{x}_{i}\left(\operatorname{var}\left(x_{i}\right)\right)^{-1}}{\sum_{i=1}^{M}\left(\operatorname{var}\left(x_{i}\right)\right)^{-1}}, \\
& \hat{y}_{\text {wa_TOA }}=\frac{\sum_{i=1}^{M} \widehat{y}_{i}\left(\operatorname{var}\left(y_{i}\right)\right)^{-1}}{\sum_{i=1}^{M}\left(\operatorname{var}\left(y_{i}\right)\right)^{-1}} .
\end{aligned}
$$

From (20) and (21), it is clear that the variance is related to the measurement error, the distance between the sensor and target and the baseline. When measurement error or the distance between the sensor and target increases, the variance increases. While when the baseline becomes longer, the variance decreases. Hence, for the proposed method, it is better to let the target be in the central range of the array and divide sensors with uniform extraction.

3.2. AOA Solution. Assume that there are $N$ anchor sensors distributed arbitrarily in a 2-D plane. We divide these sensors into $M$ groups. Each group has 2 sensors, which is the minimum number of sensors required for the AOA location algorithm. Note that some sensors may be repeated in different groups. In the $i$ th group, the $m$ th sensor is located at known position $\left(x_{i m}, y_{i m}\right)$ and the measured angles are $\widehat{\alpha}_{i m}, \quad m=1,2$, for $i=1,2, \ldots, M$. The unknown source position is located at $(x, y)$.

In $i$ th group, the true AOAs are given by [10]

$$
y-y_{i m}=\tan \alpha_{i m}\left(x-x_{i m}\right), \quad m=1,2 .
$$

Using (24), we obtain

$$
\begin{aligned}
& x=\frac{y_{i 2}-y_{i 1}+x_{i 1} \tan \alpha_{i 1}-x_{i 2} \tan \alpha_{i 2}}{\tan \alpha_{i 1}-\tan \alpha_{i 2}}, \\
& y=\frac{y_{i 2} \tan \alpha_{i 1}-y_{i 1} \tan \alpha_{i 2}+\left(x_{i 1}-x_{i 2}\right) \tan \alpha_{i 1} \tan \alpha_{i 2}}{\tan \alpha_{i 1}-\tan \alpha_{i 2}} .
\end{aligned}
$$

The AOA measurements are subject to independent zero-mean Gaussian noise

$$
\widehat{\alpha}_{i m}=\alpha_{i m}+n_{i m}, \quad m=1,2,
$$

where $n_{i m} \sim N\left(0, \sigma^{2}\right)$ and $m=1,2$. Let $\widehat{x}_{i}$ and $\hat{y}_{i}$ represent the position estimate coordinates in each group, where subscript $i$ denotes the $i$ th group, then the position estimate is 


$$
\begin{aligned}
& \widehat{x}_{i}=\frac{y_{i 2}-y_{i 1}+x_{i 1} \tan \widehat{\alpha}_{i 1}-x_{i 2} \tan \widehat{\alpha}_{i 2}}{\tan \widehat{\alpha}_{i 1}-\tan \widehat{\alpha}_{i 2}}, \\
& \widehat{y}_{i}=\frac{y_{i 2} \tan \widehat{\alpha}_{i 1}-y_{i 1} \tan \widehat{\alpha}_{i 2}+\left(x_{i 1}-x_{i 2}\right) \tan \widehat{\alpha}_{i 1} \tan \widehat{\alpha}_{i 2}}{\tan \widehat{\alpha}_{i 1}-\tan \widehat{\alpha}_{i 2}} .
\end{aligned}
$$

With 2 sensors, the target position can be solved using (27) in each group. We compute theoretical variance by using the differential method [8]. The differential of $x_{i}$ is

$$
\Delta x_{i}=\frac{\left(x_{i 1} \Delta t_{i 1}-x_{i 2} \Delta t_{i 2}\right) P_{i 1}-P_{i 3}\left(\Delta t_{i 1}-\Delta t_{i 2}\right)}{P_{i 1}^{2}},
$$

where

$$
\begin{aligned}
\Delta t_{i 1} & =\frac{\Delta \alpha_{i 1}}{\left(\cos \alpha_{i 1}\right)^{2}}, \\
\Delta t_{i 2} & =\frac{\Delta \alpha_{i 2}}{\left(\cos \alpha_{i 2}\right)^{2}},
\end{aligned}
$$

$P_{i 1}=\tan \alpha_{i 1}-\tan \alpha_{i 2}$, and $P_{i 3}=y_{i 2}-y_{i 1}+x_{i 1} \tan \alpha_{i 1}-x_{i 2}$ $\tan \alpha_{i 2}$. The differential of $y_{i}$ is

$$
\begin{aligned}
& \operatorname{var}\left(y_{i}\right)=E\left[\Delta y_{i}^{2}\right] \\
& =\frac{P_{i 1}{ }^{2}\left[y_{i 2}{ }^{2} s_{i 1}+y_{i 1}{ }^{2} s_{i 2}+\left(x_{i 1}-x_{i 2}\right)^{2}\left(t_{i 2}^{2} s_{i 1}+t_{i 1}^{2} s_{i 2}\right)\right]}{P_{i 1}^{4}} \\
& \widehat{x}_{\text {wa_AOA }}=\frac{\sum_{i=1}^{M} \widehat{x}_{i}\left(\operatorname{var}\left(x_{i}\right)\right)^{-1}}{\sum_{i=1}^{M}\left(\operatorname{var}\left(x_{i}\right)\right)^{-1}}, \\
& \widehat{y}_{\text {wa_AOA }}=\frac{\sum_{i=1}^{M} \widehat{y}_{i}\left(\operatorname{var}\left(y_{i}\right)\right)^{-1}}{\sum_{i=1}^{M}\left(\operatorname{var}\left(y_{i}\right)\right)^{-1}} .
\end{aligned}
$$$$
+\frac{2 P_{i 1}^{2}\left(y_{i 2} t_{i 2} s_{i 1}-y_{i 1} t_{i 1} s_{i 2}\right)\left(x_{i 1}-x_{i 2}\right)+P_{i 2}^{2}\left(s_{i 1}+s_{i 2}\right)-2 P_{i 1} P_{i 2}\left[y_{i 2} s_{i 1}+y_{i 1} s_{i 2}+\left(x_{i 1}-x_{i 2}\right)\left(t_{i 2} s_{i 1}-t_{i 1} s_{i 2}\right)\right]}{P_{i 1}^{4}} .
$$

The variance weighted average AOA solution is given by

Through the analysis of (31) and (33), we can obtain that when measurement error or the distance between the sensor and target increases, the variance increases. These conclusions are similar to those in TOA solution. Yet the variance increases if the baseline gets longer. Therefore, we would better group sensors with sequential extraction to get a smaller baseline in AOA solution.

\section{Performance Analysis}

In location estimation, there are two main different values that we can use to evaluate the performance of localization algorithms. One is theoretical variance, and the other one is CRLB [29, 30]. Theoretical variance is used to evaluate the practical performance of localization methods, while CRLB establishes a lower bound for any unbiased estimator. Since
$\Delta y_{i}=\frac{\left[y_{i 2} \Delta t_{i 1}-y_{i 1} \Delta t_{i 2}+\left(x_{i 1}-x_{i 2}\right)\left(\Delta t_{i 1} t_{i 2}+t_{i 1} \Delta t_{i 2}\right)\right] P_{i 1}-P_{i 2}\left(\Delta t_{i 1}-\Delta t_{i 2}\right)}{P_{i 1}^{2}}$,

where $t_{i 1}=\tan \alpha_{i 1}, \quad t_{i 2}=\tan \alpha_{i 2}, \quad$ and $P_{i 2}=y_{i 2} \tan \alpha_{i 1}-$ $y_{i 1} \tan \alpha_{i 2}+\left(x_{i 1}-x_{i 2}\right) \tan \alpha_{i 1} \tan \alpha_{i 2}$. If the angle estimation variance is $\sigma^{2}$, then the theoretical variance of $x_{i}$ is

$$
\begin{aligned}
\operatorname{var}\left(x_{i}\right) & =E\left[\Delta x_{i}^{2}\right] \\
& =\frac{P_{i 1}{ }^{2}\left(x_{i 1}{ }^{2} s_{i 1}+x_{i 2}{ }^{2} s_{i 2}\right)-\left(2 x_{i 1} s_{i 1}+2 x_{i 2} s_{i 2}\right) P_{i 1} P_{i 3}+P_{i 3}{ }^{2}\left(s_{i 1}+s_{i 2}\right)}{P_{i 1}^{4}},
\end{aligned}
$$

where

$$
\begin{aligned}
& S_{i 1}=E\left[\Delta t_{i 1}^{2}\right]=\frac{\sigma^{2}}{\left(\cos \alpha_{i 1}\right)^{4}}, \\
& S_{i 2}=E\left[\Delta t_{i 2}{ }^{2}\right]=\frac{\sigma^{2}}{\left(\cos \alpha_{i 2}\right)^{4}} .
\end{aligned}
$$

The theoretical variance of $y$ is
CRLB has already been derived in $[29,30]$, here, we derive the theoretical estimate variance of the proposed method in this section to illustrate its performance.

4.1. Theoretical Variance of TOA Solution. Assume that the variance of range measurements is $\sigma^{2}$. From (22), we can obtain the differential of $x_{\text {wa_TOA }}$ :

$$
\Delta x_{\text {wa_TOA }}=\frac{\sum_{i=1}^{M}\left(\Delta x_{i} v_{i}+x_{i} \Delta v_{i}\right) \sum_{i=1}^{M} v_{i}-\sum_{i=1}^{M} x_{i} v_{i} \sum_{i=1}^{M} \Delta v_{i}}{\left(\sum_{i=1}^{M} v_{i}\right)^{2}}
$$

where $v_{i}=\left[\operatorname{var}\left(x_{i}\right)\right]^{-1}$. Using (16) and (20), $\Delta x_{i}$ and $\Delta v_{i}$ can be written as

$$
\begin{aligned}
& \Delta x_{i}=\left(a_{i 3}-a_{i 2}\right) d_{i 1} \Delta d_{i 1}+a_{i 2} d_{i 2} \Delta d_{i 2}-a_{i 3} d_{i 3} \Delta d_{i 3}, \\
& \Delta v_{i}=t_{i 1} \Delta d_{i 1}+t_{i 2} \Delta d_{i 2}+t_{i 3} \Delta d_{i 3},
\end{aligned}
$$

where $t_{i 1}=-2\left[\operatorname{var}\left(x_{i}\right)\right]^{-2}\left(a_{i 3}-a_{i 2}\right)^{2} d_{i 1} \sigma^{2}, t_{i 2}=-2\left[\operatorname{var}\left(x_{i}\right)\right]^{-2}$ $a_{i 2}{ }^{2} d_{i 2} \sigma^{2}$, and $t_{i 3}=-2\left[\operatorname{var}\left(x_{i}\right)\right]^{-2} a_{i 3}{ }^{2} d_{i 3} \sigma^{2}$. Then, we have

$$
\Delta x_{i} v_{i}+x_{i} \Delta v_{i}=k_{i 1} \Delta d_{i 1}+k_{i 2} \Delta d_{i 2}+k_{i 3} \Delta d_{i 3},
$$


where $k_{i 1}=v_{i}\left(a_{i 3}-a_{i 2}\right) d_{i 1}+x_{i} t_{i 1}, \quad k_{i 2}=v_{i} a_{i 2} d_{i 2}+x_{i} t_{i 2}$, and $k_{i 3}=-v_{i} a_{i 3} d_{i 3}+x_{i} t_{i 3}$.
Substituting (37), (38), and (39) into (36), the theoretical estimate variance of $x_{\text {wa_TOA }}$ is

$$
\begin{aligned}
\operatorname{var}\left(x_{\text {wa_TOA }}\right)= & E\left[\Delta x_{\text {wa_TOA }}{ }^{2}\right] \\
= & \frac{\sigma^{2}\left(\sum_{i=1}^{M} v_{i}\right)^{2} \sum_{i=1}^{M}\left(k_{i 1}{ }^{2}+k_{i 2}{ }^{2}+k_{i 3}{ }^{2}\right)+\sigma^{2}\left(\sum_{i=1}^{M} x_{i} v_{i}\right)^{2} \sum_{i=1}^{M}\left(t_{i 1}{ }^{2}+t_{i 2}{ }^{2}+t_{i 3}{ }^{2}\right)}{\left(\sum_{i=1}^{M} v_{i}\right)^{4}} \\
& -\frac{2 \sigma^{2}\left(\sum_{i=1}^{M} v_{i}\right)\left(\sum_{i=1}^{M} x_{i} v_{i}\right) \sum_{i=1}^{M}\left(k_{i 1} t_{i 1}+k_{i 2} t_{i 2}+k_{i 3} t_{i 3}\right)}{\left(\sum_{i=1}^{M} v_{i}\right)^{4}} .
\end{aligned}
$$

With the similar derivation, the theoretical estimate variance of $y_{\text {wa_TOA }}$ is

$$
\begin{aligned}
\operatorname{var}\left(y_{\text {wa_tOA }}\right)= & E\left[\Delta y_{\text {wa_TOA }}{ }^{2}\right] \\
= & \frac{\sigma^{2}\left(\sum_{i=1}^{M} w_{i}\right)^{2} \sum_{i=1}^{M}\left(j_{i 1}{ }^{2}+j_{i 2}{ }^{2}+j_{i 3}{ }^{2}\right)+\sigma^{2}\left(\sum_{i=1}^{M} y_{i} w_{i}\right)^{2} \sum_{i=1}^{M}\left(s_{i 1}{ }^{2}+s_{i 2}{ }^{2}+s_{i 3}{ }^{2}\right)}{\left(\sum_{i=1}^{M} w_{i}\right)^{4}} \\
& -\frac{2 \sigma^{2}\left(\sum_{i=1}^{M} w_{i}\right)\left(\sum_{i=1}^{M} y_{i} w_{i}\right) \sum_{i=1}^{M}\left(j_{i 1} s_{i 1}+j_{i 2} s_{i 2}+j_{i 3} s_{i 3}\right)}{\left(\sum_{i=1}^{M} w_{i}\right)^{4}}
\end{aligned}
$$

where $w_{i}=\left[\operatorname{var}\left(y_{i}\right)\right]^{-1}, \quad s_{i 1}=-2\left[\operatorname{var}\left(y_{i}\right)\right]^{-2}\left(b_{i 3}-b_{i 2}\right)^{2} d_{i 1} \sigma^{2}$, $s_{i 2}=-2\left[\operatorname{var}\left(y_{i}\right)\right]^{-2} b_{i 2}{ }^{2} d_{i 2} \sigma^{2}, s_{i 3}=-2\left[\operatorname{var}\left(y_{i}\right)\right]^{-2} b_{i 3}{ }^{2} d_{i 3} \sigma^{2}, j_{i 1}$ $=w_{i}\left(\mathrm{~b}_{i 3}-b_{i 2}\right) d_{i 1}+y_{i} s_{i 1}, j_{i 2}=w_{i} b_{i 2} d_{i 2}+y_{i} s_{i 2}$, and $j_{i 3}=-w_{i}$ $b_{i 3} d_{i 3}+y_{i} s_{i 3}$.

The theoretical estimate variance of the proposed method is then obtained from (40) and (41) as

$$
\operatorname{var}_{\text {wa_TOA }}=\operatorname{var}\left(x_{\text {wa_TOA }}\right)+\operatorname{var}\left(y_{\text {wa_TOA }}\right) .
$$

Figure 3 depicts the theoretical variance of the proposed method in the TOA case as the target position varies, where the sensor positions are $\left(x_{1}=0, y_{1}=1000\right),\left(x_{2}=1000 \mathrm{sin}\right.$ $\left.40^{\circ}, y_{2}=1000 \cos 40^{\circ}\right),\left(x_{3}=1000 \sin 80^{\circ}, y_{3}=1000 \cos 80^{\circ}\right)$, $\left(x_{4}=1000 \sin 60^{\circ}, y_{4}=-1000 \cos 60^{\circ}\right), \quad\left(x_{5}=1000 \sin 20^{\circ}\right.$, $\left.y_{5}=-1000 \cos 20^{\circ}\right),\left(x_{6}=-1000 \sin 20^{\circ}, y_{6}=-1000 \cos 20^{\circ}\right)$, $\left(x_{7}=-1000 \sin 60^{\circ}, y_{7}=-1000 \cos 60^{\circ}\right),\left(x_{8}=-1000 \sin 80^{\circ}\right.$, $\left.y_{8}=1000 \cos 80^{\circ}\right)$, and $\left(x_{9}=-1000 \sin 40^{\circ}, y_{9}=1000 \cos \right.$ $\left.40^{\circ}\right)$ and $\sigma_{\mathrm{TOA}}$ is fixed at 50 . It shows that the estimation is more accurate when the target gets closer to the central of the array range.

4.2. Theoretical Variance of AOA Solution. Assume that the variance of angle measurements is $\sigma^{2}$. From (28), $\Delta x_{i}$ can be written as

$$
\Delta x_{i}=R_{i 1} \Delta \alpha_{i 1}+R_{i 2} \Delta \alpha_{i 2}
$$

where

$$
\begin{aligned}
R_{i 1} & =\frac{x_{i 1} /\left(\cos \alpha_{i 1}\right)^{2}\left(\tan \alpha_{i 1}-\tan \alpha_{i 2}\right)-\left(y_{i 2}-y_{i 1}+x_{i 1} \tan \alpha_{i 1}-x_{i 2} \tan \alpha_{i 2}\right)\left(1 /\left(\cos \alpha_{i 1}\right)^{2}\right)}{\left(\tan \alpha_{i 1}-\tan \alpha_{i 2}\right)^{2}}, \\
R_{i 2} & =\frac{-\left(x_{i 2} /\left(\cos \alpha_{i 2}\right)^{2}\right)\left(\tan \alpha_{i 1}-\tan \alpha_{i 2}\right)+\left(y_{i 2}-y_{i 1}+x_{i 1} \tan \alpha_{i 1}-x_{i 2} \tan \alpha_{i 2}\right)\left(1 /\left(\cos \alpha_{i 2}\right)^{2}\right)}{\left(\tan \alpha_{i 1}-\tan \alpha_{i 2}\right)^{2}} .
\end{aligned}
$$




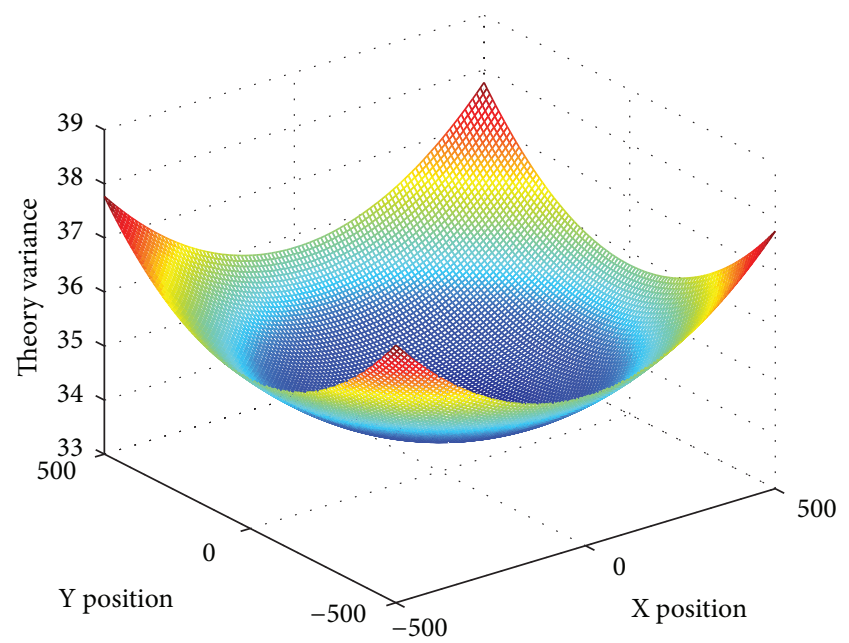

FIGURE 3: The theoretical variance of the proposed method in TOA case as the target position varies.

From (34), we can obtain the differential of $x_{\text {wa_AOA }}$

$$
\Delta x_{\text {wa } \_ \text {AOA }}=\frac{\sum_{i=1}^{M}\left(\Delta x_{i} v_{i}+x_{i} \Delta v_{i}\right) \sum_{i=1}^{M} v_{i}-\sum_{i=1}^{M} x_{i} v_{i} \sum_{i=1}^{M} \Delta v_{i}}{\left(\sum_{i=1}^{M} v_{i}\right)^{2}},
$$

where $v_{i}=\left[\operatorname{var}\left(x_{i}\right)\right]^{-1}$. Using (31), $\Delta v_{i}$ can be written as

$$
\Delta v_{i}=\mathrm{C}_{i 1} \Delta \alpha_{i 1}+C_{i 2} \Delta \alpha_{i 2}
$$

where

$$
\begin{aligned}
\operatorname{var}\left(x_{\text {wa_AOA }}\right) & =E\left[\Delta x_{\text {wa_AOA }}{ }^{2}\right] \\
& =\frac{\sigma^{2}\left(\sum_{i=1}^{M} v_{i}\right)^{2} \sum_{i=1}^{M}\left(B_{i 1}{ }^{2}+B_{i 2}{ }^{2}\right)+\sigma^{2}\left(\sum_{i=1}^{M} x_{i} v_{i}\right)^{2} \sum_{i=1}^{M}\left(C_{i 1}{ }^{2}+C_{i 2}{ }^{2}\right)-2 \sigma^{2}\left(\sum_{i=1}^{M} v_{i}\right)\left(\sum_{i=1}^{M} x_{i} v_{i}\right) \sum_{i=1}^{M}\left(B_{i 1} C_{i 1}+B_{i 2} C_{i 2}\right)}{\left(\sum_{i=1}^{M} v_{i}\right)^{4}} .
\end{aligned}
$$

With the similar derivation, the theoretical estimate variance of $y_{\text {wa_AOA }}$ is

$$
\begin{aligned}
\operatorname{var}\left(y_{\text {wa_AOA }}\right) & =E\left[\Delta y_{\text {wa_AOA }}{ }^{2}\right] \\
& =\frac{\sigma^{2}\left(\sum_{i=1}^{M} w_{i}\right)^{2} \sum_{i=1}^{M}\left(G_{i 1}{ }^{2}+G_{i 2}{ }^{2}\right)+\sigma^{2}\left(\sum_{i=1}^{M} y_{i} w_{i}\right)^{2} \sum_{i=1}^{M}\left(E_{i 1}{ }^{2}+E_{i 2}{ }^{2}\right)}{\left(\sum_{i=1}^{M} w_{i}\right)^{4}}-\frac{2 \sigma^{2}\left(\sum_{i=1}^{M} w_{i}\right)\left(\sum_{i=1}^{M} y_{i} w_{i}\right) \sum_{i=1}^{M}\left(G_{i 1} E_{i 1}+G_{i 2} E_{i 2}\right)}{\left(\sum_{i=1}^{M} w_{i}\right)^{4}},
\end{aligned}
$$


where

$$
\begin{aligned}
& w_{i}=\left[\operatorname{var}\left(y_{i}\right)\right]^{-1}, \\
& G_{i 1}=w_{i} J_{i 1}+y_{i} E_{i 1} \text {, } \\
& G_{i 2}=w_{i} J_{i 2}+y_{i} E_{i 2} \text {, } \\
& E_{i 1}=-\left[\operatorname{var}\left(y_{i}\right)\right]^{-2} \cdot \frac{1}{P_{i 1}^{8}} \cdot\left[\frac{f_{i 1}}{\left(\cos \alpha_{i 1}\right)^{2}}+\frac{f_{i 2} y_{i 2}}{\left(\cos \alpha_{i 1}\right)^{2}}+\frac{f_{i 2}\left(x_{i 1}-x_{i 2}\right) \tan \alpha_{i 2}}{\left(\cos \alpha_{i 1}\right)^{2}}+\frac{4 \sigma^{2} f_{i 3}}{\left(\cos \alpha_{i 1}\right)^{5}} \sin \alpha_{i 1}+\frac{f_{i 5}}{\left(\cos \alpha_{i 1}\right)^{2}}\right] \text {, } \\
& E_{i 2}=-\left[\operatorname{var}\left(y_{i}\right)\right]^{-2} \cdot \frac{1}{P_{i 1}^{8}} \cdot\left[\frac{f_{i 1}}{\left(\cos \alpha_{i 2}\right)^{2}}-\frac{f_{i 2} y_{i 2}}{\left(\cos \alpha_{i 2}\right)^{2}}+\frac{f_{i 2}\left(x_{i 1}-x_{i 2}\right) \tan \alpha_{i 1}}{\left(\cos \alpha_{i 2}\right)^{2}}+\frac{4 \sigma^{2} f_{i 4}}{\left(\cos \alpha_{i 2}\right)^{5}} \sin \alpha_{i 1}+\frac{f_{i 6}}{\left(\cos \alpha_{i 2}\right)^{2}}\right] \text {, } \\
& f_{i 1}=P_{i 1}^{4}\left(2 P_{i 1} q_{i 1}-2 P_{i 2} q_{i 2}\right)-4 q_{i 3} P_{i 1}^{3} \text {, } \\
& f_{i 2}=P_{i 1}^{4}\left[2 P_{i 2}\left(s_{i 1}+s_{i 2}\right)-2 P_{i 1} q_{i 2}\right] \text {, } \\
& f_{i 3}=P_{i 1}^{4}\left\{P_{i 1}^{2}\left[y_{i 2}^{2}+\left(x_{i 1}-x_{i 2}\right)^{2} t_{i 2}^{2}+2\left(x_{i 1}-x_{i 2}\right) y_{i 2} t_{i 2}\right]+P_{i 2}^{2}-2 P_{i 1} P_{i 2}\left[y_{i 2}+\left(x_{i 1}-x_{i 2}\right) t_{i 2}\right]\right\} \text {, } \\
& f_{i 4}=P_{i 1}^{4}\left\{P_{i 1}^{2}\left[y_{i 1}^{2}+\left(x_{i 1}-x_{i 2}\right)^{2} t_{i 1}^{2}-2\left(x_{i 1}-x_{i 2}\right) y_{i 1} t_{i 1}\right]+P_{i 2}^{2}-2 P_{i 1} P_{i 2}\left[y_{i 1}-\left(x_{i 1}-x_{i 2}\right) t_{i 1}\right]\right\} \text {, } \\
& f_{i 5}=P_{i 1}^{4}\left\{P_{i 1}^{2}\left[2\left(x_{i 1}-x_{i 2}\right)^{2} t_{i 1} S_{i 2}-2\left(x_{i 1}-x_{i 2}\right) y_{i 1} S_{i 2}\right]-2 P_{i 1} P_{i 2}\left[-\left(x_{i 1}-x_{i 2}\right) S_{i 2}\right]\right\} \text {, } \\
& f_{i 6}=P_{i 1}^{4}\left\{P_{i 1}^{2}\left[2\left(x_{i 1}-x_{i 2}\right)^{2} t_{i 2} S_{i 1}+2\left(x_{i 1}-x_{i 2}\right) y_{i 2} S_{i 1}\right]-2 P_{i 1} P_{i 2}\left[\left(x_{i 1}-x_{i 2}\right) S_{i 1}\right]\right\} \text {, } \\
& q_{i 1}=y_{i 2}{ }^{2} s_{i 1}+y_{i 1}{ }^{2} s_{i 2}+\left(x_{i 1}-x_{i 2}\right)^{2}\left(t_{i 2}^{2} s_{i 1}+t_{i 1}^{2} s_{i 2}\right)+2\left(y_{i 2} t_{i 2} s_{i 1}-y_{i 1} t_{i 1} s_{i 2}\right)\left(x_{i 1}-x_{i 2}\right), \\
& q_{i 2}=y_{i 2} s_{i 1}+y_{i 1} s_{i 2}+\left(x_{i 1}-x_{i 2}\right)\left(t_{i 2} s_{i 1}-t_{i 1} s_{i 2}\right) \text {, } \\
& q_{i 3}=P_{i 1}{ }^{2}\left[y_{i 2}{ }^{2} s_{i 1}+y_{i 1}{ }^{2} s_{i 2}+\left(x_{i 1}-x_{i 2}\right)^{2}\left(t_{i 2}^{2} s_{i 1}+t_{i 1}^{2} s_{i 2}\right)\right]+2 P_{i 1}{ }^{2}\left(y_{i 2} t_{i 2} s_{i 1}-y_{i 1} t_{i 1} s_{i 2}\right)\left(x_{i 1}-x_{i 2}\right)+P_{i 2}{ }^{2}\left(s_{i 1}+s_{i 2}\right) \\
& -2 P_{i 1} P_{i 2}\left[y_{i 2} s_{i 1}+y_{i 1} s_{i 2}+\left(x_{i 1}-x_{i 2}\right)\left(t_{i 2} s_{i 1}-t_{i 1} s_{i 2}\right)\right] \text {, } \\
& J_{i 1}=\frac{\left[\left(y_{i 2} /\left(\cos \alpha_{i 1}\right)^{2}\right)+\left(\left(x_{i 1}-x_{i 2}\right) \tan \alpha_{i 2} /\left(\cos \alpha_{i 1}\right)^{2}\right)\right] P_{i 1}-\left(P_{i 2} /\left(\cos \alpha_{i 1}\right)^{2}\right)}{P_{i 1}^{2}}, \\
& J_{i 2}=\frac{\left[-\left(y_{i 1} /\left(\cos \alpha_{i 2}\right)^{2}\right)+\left(\left(x_{i 1}-x_{i 2}\right) \tan \alpha_{i 1} /\left(\cos \alpha_{i 2}\right)^{2}\right)\right] P_{i 1}+\left(P_{i 2} /\left(\cos \alpha_{i 2}\right)^{2}\right)}{P_{i 1}^{2}} \text {. }
\end{aligned}
$$

The theoretical estimate variance of the proposed method is then obtained from (49) and (50) as

$$
\operatorname{var}_{\text {wa_AOA }}=\operatorname{var}\left(x_{\text {wa_AOA }}\right)+\operatorname{var}\left(y_{\text {wa_AOA }}\right) \text {. }
$$

The theoretical variance of AOA solution is illustrated in Figure 4 as the target position varies, where the sensor positions are $\left(x_{1}=-1000, y_{1}=1000\right),\left(x_{2}=1000, y_{2}=1000\right)$, $\left(x_{3}=1000, y_{3}=-1000\right)$, and $\left(x_{4}=-1000, y_{4}=-1000\right)$ and $\sigma_{\mathrm{AOA}}$ is fixed at $3^{\circ}$. The result is similar to TOA solution that the proposed method can give a more accurate estimation if the target gets closer to the central of the array range.

\section{Simulation Results}

Simulations are performed to validate the performance of the proposed method and to compare the relative localization accuracy for different methods.

Without loss of generality, we assume that the signal and noises are mutually independent, zero-mean stationary
Gaussian random processes. Suppose that $\sigma_{\mathrm{TOA}}$ and $\sigma_{\mathrm{AOA}}$ denote TOA standard deviations and AOA standard deviations, respectively. Simulation results are displayed in terms of the root-mean-square error (RMSE) given by $\sqrt{E\left[\left(x_{\mathrm{es}}-x\right)^{2}+\left(y_{e s}-y\right)^{2}\right]}[15]$ which are obtained from the average of 3000 independent runs.

As shown in Figures 5(a) and 5(b), the correctness of the theoretical variance we derive is verified in TOA solution and AOA solution, respectively. The source is at an arbitrary position within the range of the array. The Monte Carlo experiment generates a source position randomly in each run. Simulations demonstrated that the theoretical variance fits well with RMSE in both TOA solution and AOA solution.

Figure 6(a) illustrates the performance of the proposed method as the number of the sensor varies, where the area size is $1000, \sigma_{\mathrm{TOA}}$ is fixed at 30 , and the source is at $(x=-200, y=-100)$. The proposed method with the simplified formulae instead of matrix inversion still 


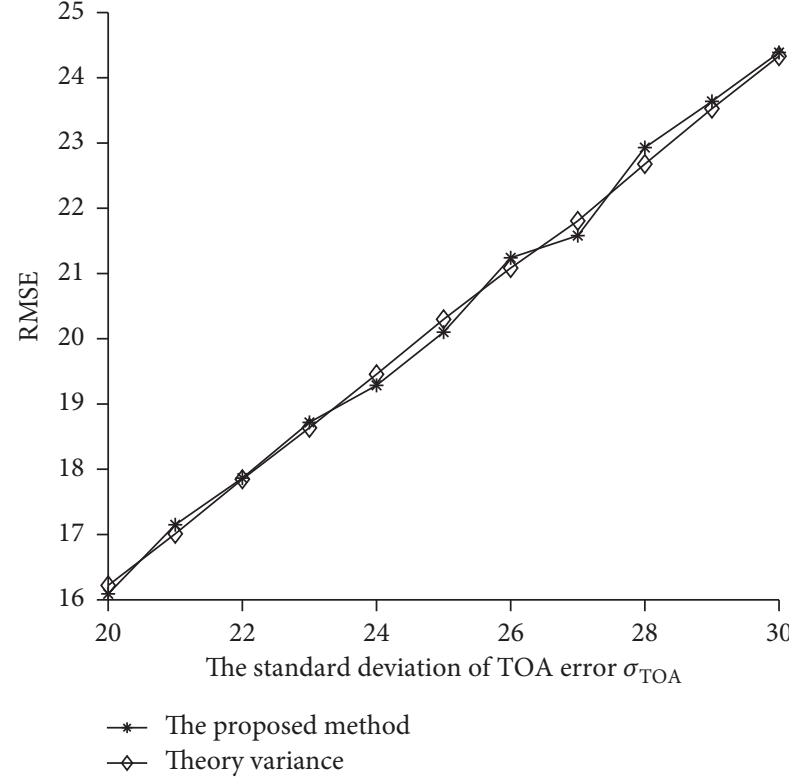

(a)

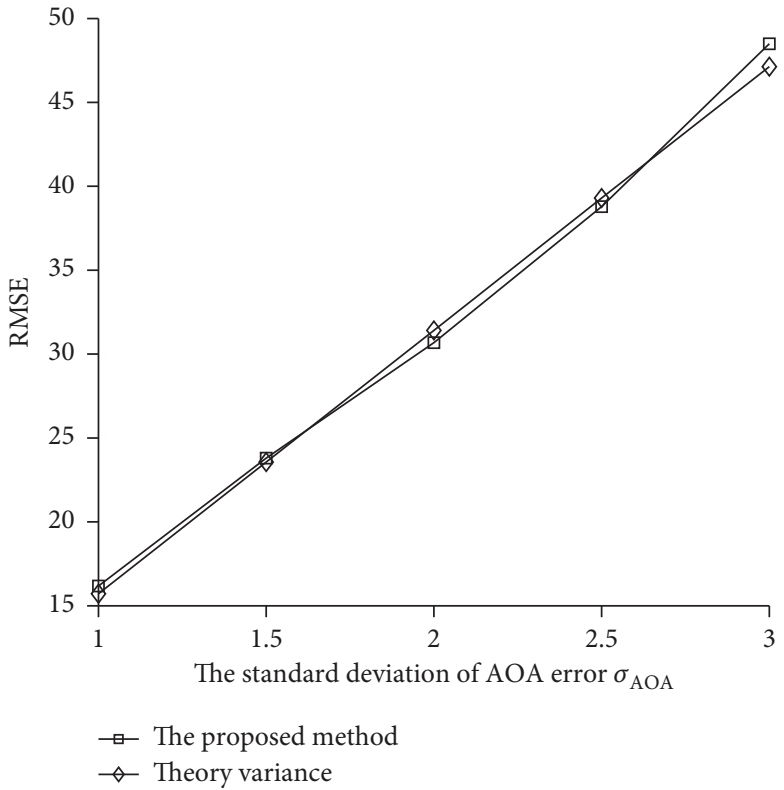

(b)

FIGURE 5: The theoretical variance and RMSE of the proposed method: (a) theoretical variance in TOA solution with 9 sensors as $\sigma_{\text {TOA }}$ varies; (b) theoretical variance in AOA solution with 8 sensors as $\sigma_{\mathrm{AOA}}$ varies.

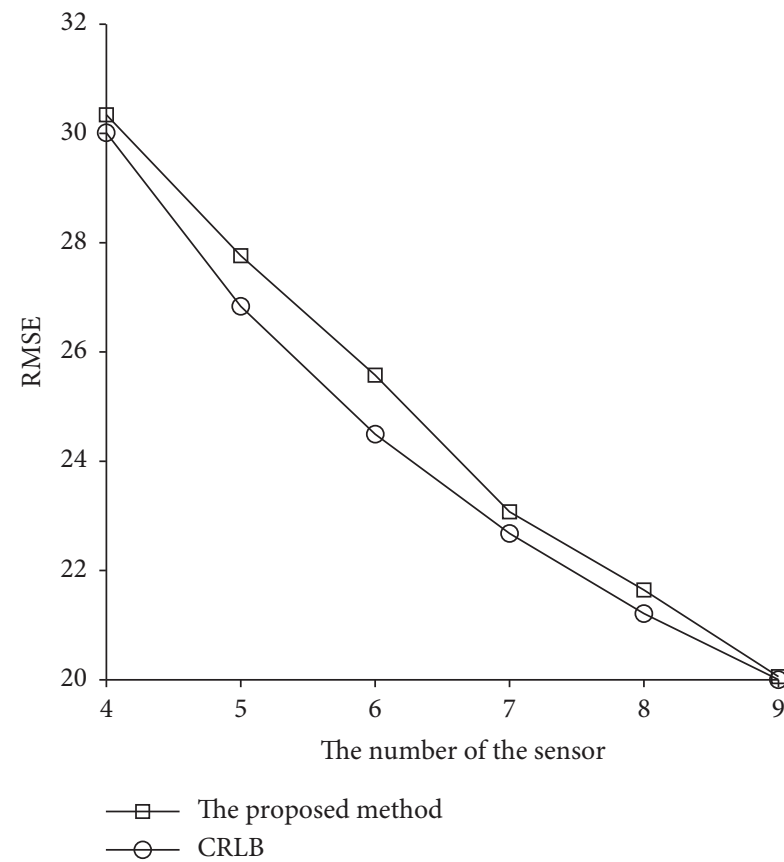

(a)

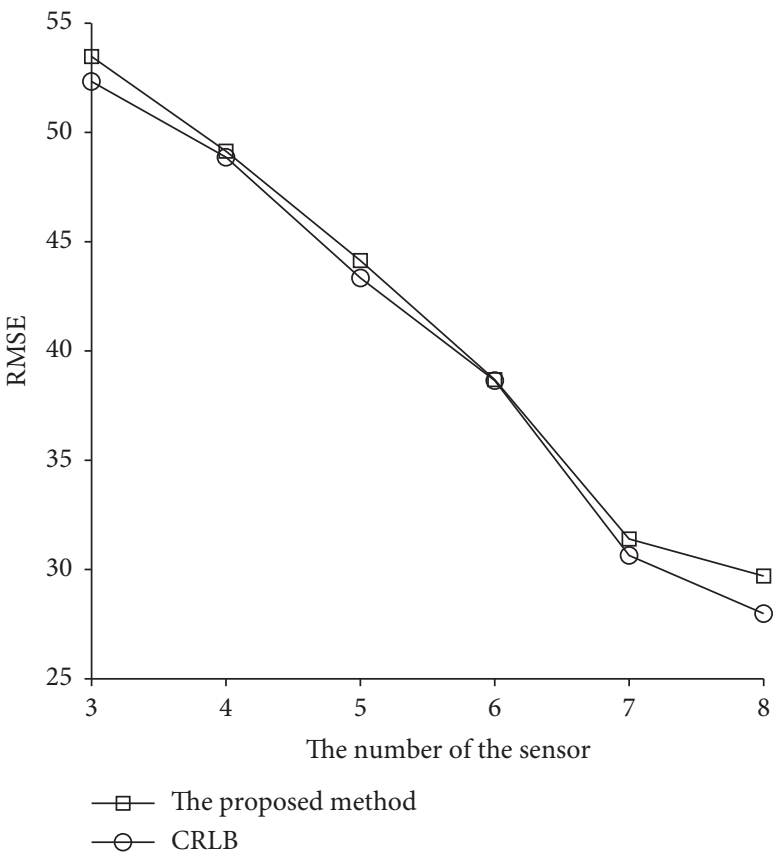

(b)

FIGURE 6: Performance of the proposed method as sensor number varies: (a) RMSE of TOA solution ( $\sigma_{\text {TOA }}$ is fixed at 30 and the source is at $(x=-200, y=-100))$; (b) RMSE of AOA solution $\left(\sigma_{\mathrm{AOA}}\right.$ is fixed at $2^{\circ}$ and the source is at $\left.(x=100, y=-200)\right)$.

performs well, and its localization accuracy is close to CRLB. In case of AOA solution, the results are given in Figure 6(b), where the area size is $1000, \sigma_{\mathrm{AOA}}$ is fixed at $2^{\circ}$, and the source is at $(x=100, y=-200)$. The performance of the proposed method also performs well in AOA solution.
In order to verify dynamic localization performance of the proposed method, simulations of dynamic positioning were carried out. Figures $7(\mathrm{a})$ and $7(\mathrm{~b})$ compare the real target trace and estimated target trace in TOA solution. Two different motion trajectories of $W$ and $S$ are simulated in Figures $7(\mathrm{a})$ and $7(\mathrm{~b})$, respectively. $\sigma_{\mathrm{TOA}}$ is fixed at 40 . 


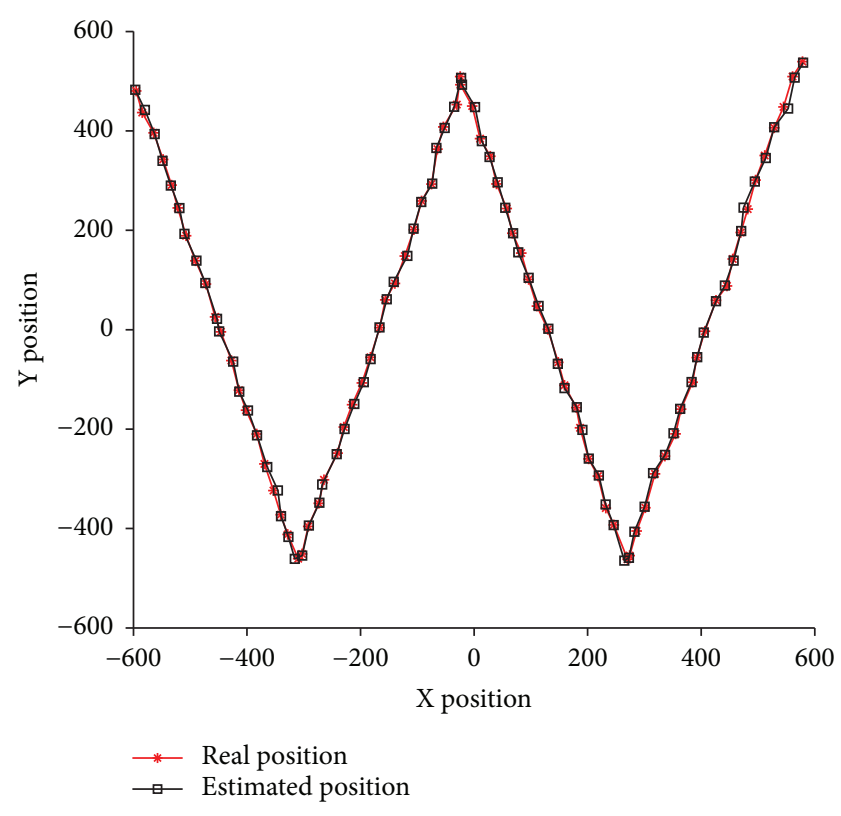

(a)

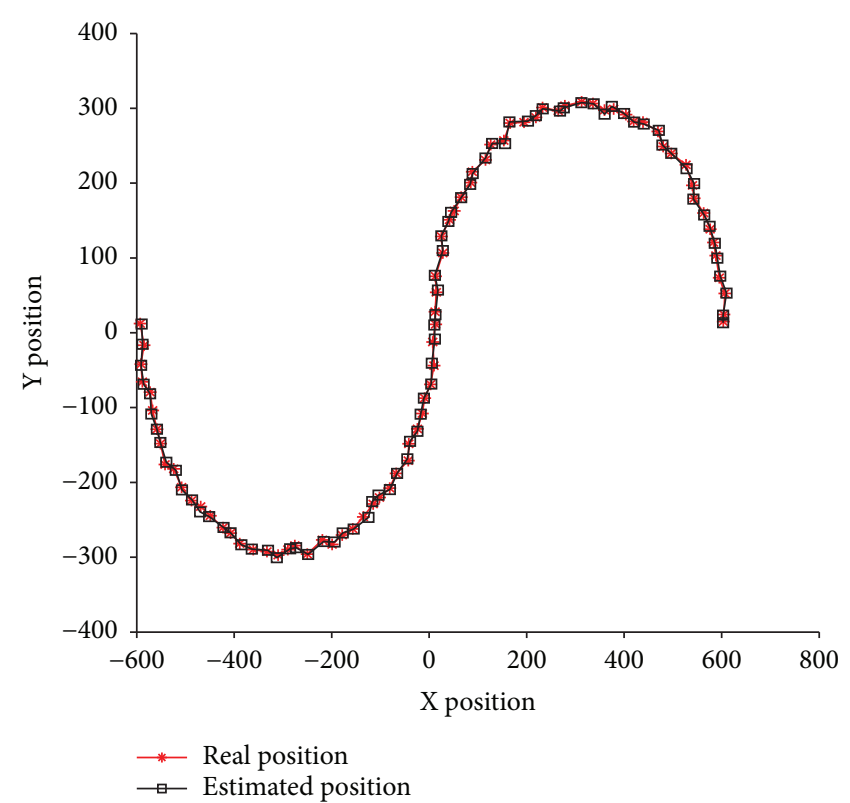

(b)

Figure 7: Dynamic localization performance of the proposed method in TOA solution: (a) the comparison between real target trace and estimated target trace in $W$ trajectory; (b) the comparison between real target trace and estimated target trace in $S$ trajectory.

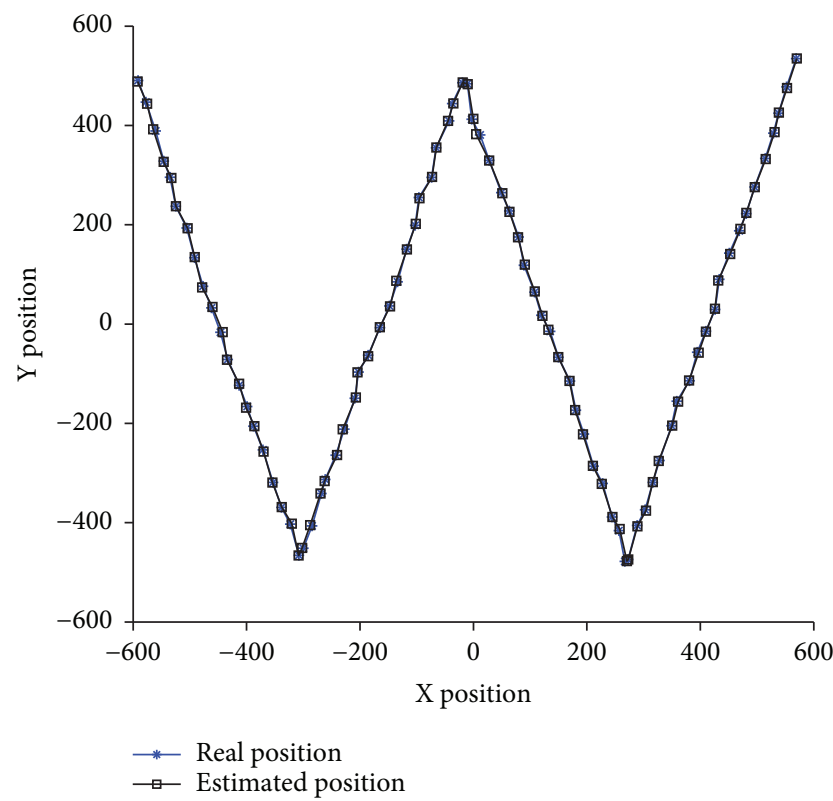

(a)

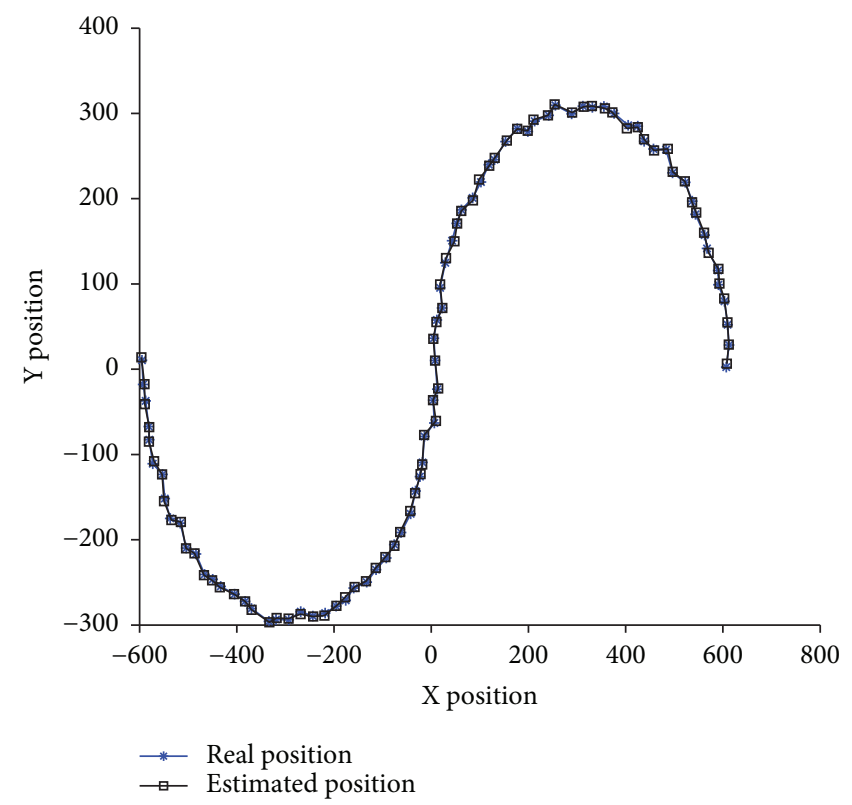

(b)

FIGURE 8: Dynamic localization performance of the proposed method in AOA solution: (a) the comparison between real target trace and estimated target trace in $W$ trajectory; (b) the comparison between real target trace and estimated target trace in $S$ trajectory.

Both Figures 7(a) and 7(b) show that the proposed method performs well in dynamic localization. The real target trace and the estimated target trace are almost coincident. Similar simulation was conducted in AOA solution as shown in Figures $8(\mathrm{a})$ and $8(\mathrm{~b})$, where $\sigma_{\mathrm{AOA}}$ is fixed at $2^{\circ}$.

Figures 8(a) and 8(b) show that the AOA solution of the proposed method achieves high accuracy in dynamic localization.
To give a comparison of the localization accuracy between the proposed method and other methods, Figure 9(a) compares the performance of the proposed method, Chan's method [8], and direct average method in TOA solution with 4 sensors. The sensor positions are $\left(x_{1}=-1000, y_{1}=1000\right),\left(x_{2}=1000, y_{2}=1000\right),\left(x_{3}=1000\right.$, $\left.y_{3}=-1000\right)$, and $\left(x_{4}=-1000, y_{4}=-1000\right)$. The source is at an arbitrary position within the range of the array. 


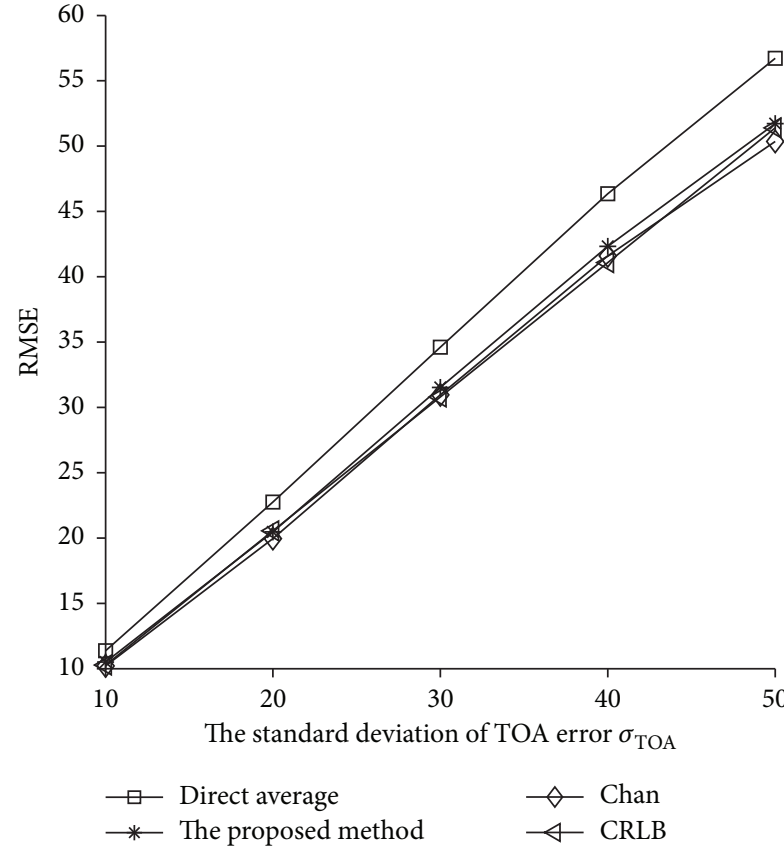

(a)

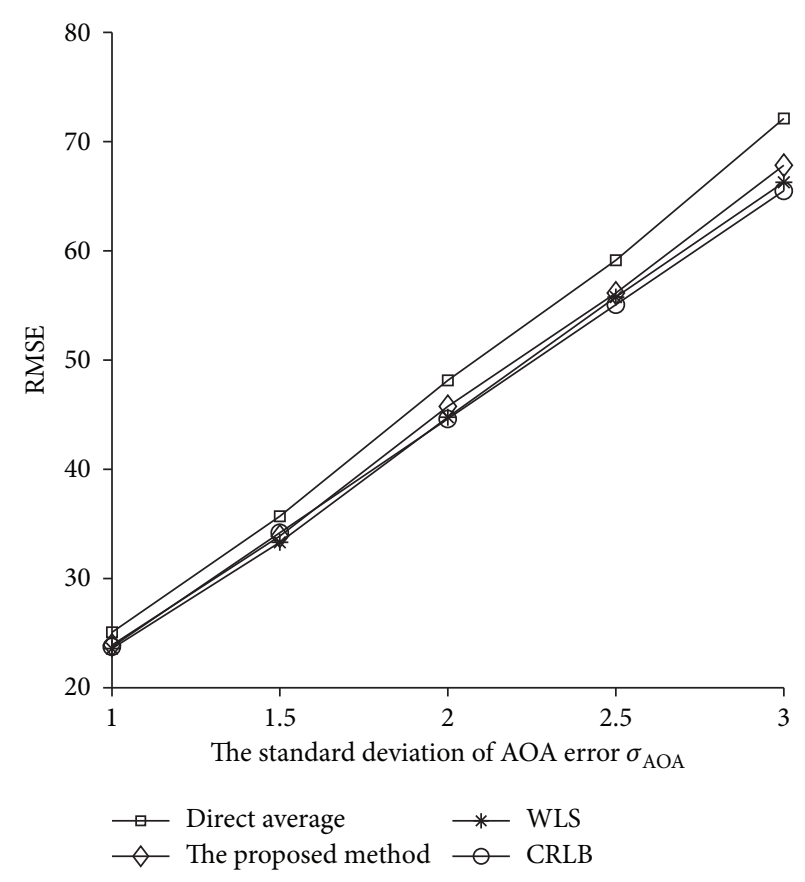

(b)

FIgURE 9: Performance comparison with other methods and CRLB: (a) RMSE of the direct average method, the proposed method, and the Chan's method in TOA solution as $\sigma_{\text {TOA }}$ varies; (b) RMSE of the direct average method, the proposed method, and the WLS method in AOA solution as $\sigma_{\mathrm{AOA}}$ varies.

The Monte Carlo experiment generates a source position randomly in each run. Simulations show the estimation performance of the proposed method as $\sigma_{\text {TOA }}$ varies from 10 to 50. Among the three methods, the direct average method performs worst and the proposed method gives a smaller RMSE using variance weighting. The positioning accuracy of the proposed method is close to the Chan's method and CRLB [29]. Note that no matrix reverse is required for the proposed method; the computation complexity is largely simplified, and the method can be used in low-cost hardware devices.

Similar experiment was conducted in AOA solution as shown in Figure 9(b). The sensor positions are $\left(x_{1}=0\right.$, $\left.y_{1}=0\right), \quad\left(x_{2}=1000, y_{2}=1000\right), \quad\left(x_{3}=1000, y_{3}=1000\right)$, and $\left(x_{4}=0, y_{4}=1000\right)$. Simulations show the localization accuracy of the proposed method compared with the WLS method [11] and direct average method as $\sigma_{\mathrm{AOA}}$ varies from $1^{\circ}$ to $3^{\circ}$. The performance of the proposed method remains promising in AOA solution.

As seen in all the figures, the proposed method using theoretical variances as weights to improve the system performance provides higher accuracy than the method that uses the direct average of each group's estimate result. Previous closed-form solutions cannot be used for low-cost hardware devices due to matrix inversion. This method, on the other hand, provides an effective solution which has the similar performance with the matrix method for target localization with low-cost hardware devices.

\section{Conclusions}

We have developed a simple method for target localization in a multistation redundancy system. The proposed method, which utilizes groups to avoid matrix inversion and uses theoretical variances as weights to improve the system performance, gives an algebraic solution which can be applied to low-cost hardware devices. It requires less computation and does not reply on matrix inversion. The performance of the proposed method is verified by the simulation results. A comparison with other localization methods and CRLB was conducted. With theoretical variances used as the weighting coefficients, the proposed method has a significantly reduced RMSE compared with the method that directly averages each group's localization result. The positioning accuracy of the proposed method is close to CRLB both in TOA solution and AOA solution.

This paper introduces the variance weighted average method for target localization in a multistation redundancy system. The proposed method, without matrix inversion, has the similar performance with the matrix method. For many low-cost hardware devices applications, this closedform solution may itself be sufficiently accurate.

\section{Data Availability}

All data generated or analysed during this study are included in this paper. No additional data are available. 


\section{Conflicts of Interest}

The authors declare that there is no conflict of interest regarding the publication of this paper.

\section{Acknowledgments}

The work undertaken in this paper was supported by the Fundamental Research Funds for the Central Universities of China (ZYGX2016J030).

\section{References}

[1] J. M. Fresno, G. Robles, J. M. Martínez-Tarifa, and B. G. Stewart, "Survey on the performance of source localization algorithms," Sensors, vol. 17, no. 11, p. 2666, 2017.

[2] J. C. K. Chow, M. Peter, M. Scaioni, and M. Al-Durgham, "Indoor tracking, mapping, and navigation: algorithms, technologies, and applications," Journal of Sensors, vol. 2018, Article ID 5971752, 3 pages, 2018.

[3] F. Mekelleche and H. Haffaf, "Classification and comparison of range-based localization techniques in wireless sensor networks," Journal of Communications, vol. 12, 2017.

[4] A. M. A. Abu Znaid, M. Y. I. Idris, A. W. Abdul Wahab, L. Khamis Qabajeh, and O. Adil Mahdi, "Sequential Monte Carlo localization methods in mobile wireless sensor networks: a review," Journal of Sensors, vol. 2017, Article ID 1430145, 19 pages, 2017.

[5] C. Wang, F. Qi, G. Shi, and J. Ren, "A linear combinationbased weighted least square approach for target localization with noisy range measurements," Signal Processing, vol. 94, pp. 202-211, 2014.

[6] J. J. Caffery Jr., "A new approach to the geometry of TOA location," in Vehicular Technology Conference Fall 2000. IEEE VTS Fall VTC2000. 52nd Vehicular Technology Conference (Cat. No. 00CH37152), pp. 1943-1949, Boston, MA, USA, September 2002.

[7] N. H. Nguyen and K. Doğançay, "Optimal geometry analysis for multistatic TOA localization," IEEE Transactions on Signal Processing, vol. 64, no. 16, pp. 4180-4193, 2016.

[8] Y. T. Chan and K. C. Ho, "A simple and efficient estimator for hyperbolic location," IEEE Transactions on Signal Processing, vol. 42, no. 8, pp. 1905-1915, 1994.

[9] I. O. Tovkach and S. Y. Zhuk, "Recurrent algorithm for TDOA localization in sensor networks," Journal of Aerospace Technology and Management, vol. 9, no. 4, pp. 489-494, 2017.

[10] M. Gavish and A. J. Weiss, "Performance analysis of bearingonly target location algorithms," IEEE Transactions on Aerospace and Electronic Systems, vol. 28, no. 3, pp. 817-828, 1992.

[11] K. Doğançay, "Bearings-only target localization using total least squares," Signal Processing, vol. 85, no. 9, pp. 16951710, 2005.

[12] S. C. Nardone and M. L. Graham, "A closed-form solution to bearings-only target motion analysis," IEEE Journal of Oceanic Engineering, vol. 22, no. 1, pp. 168-178, 1997.

[13] F. Seco and A. Jiménez, "Smartphone-based cooperative indoor localization with RFID technology," Sensors, vol. 18, no. 1, p. 266, 2018.

[14] A. N. Bishop and P. Jensfelt, "An optimality analysis of sensortarget geometries for signal strength based localization," in 2009 International Conference on Intelligent Sensors, Sensor
Networks and Information Processing (ISSNIP), pp. 127-132, Melbourne, VIC, Australia, December 2009.

[15] T. L. N. Nguyen and Y. Shin, "Matrix completion optimization for localization in wireless sensor networks for intelligent IoT," Sensors, vol. 16, no. 5, p. 722, 2016.

[16] Y. Miao, H. Wu, and L. Zhang, "The accurate location estimation of sensor node using received signal strength measurements in large-scale farmland," Journal of Sensors, vol. 2018, Article ID 2325863, 10 pages, 2018.

[17] J. Yin, Q. Wan, S. Yang, and K. C. Ho, "A simple and accurate TDOA-AOA localization method using two stations," IEEE Signal Processing Letters, vol. 23, no. 1, pp. 144-148, 2016.

[18] W. Shi, X. Qi, J. Li et al., "Simple solution to the optimal deployment of cooperative nodes in two-dimensional TOA-based and AOA-based localization system," Eurasip Journal on Wireless Communications and Networking, vol. 2017, no. 1, p. 79, 2017.

[19] J. J. Caffery, Wireless Location in CDMA Cellular Radio Systems, Vol. 535, Kluwer Academic Publishers, 1999.

[20] G. Mao, B. Fidan, and B. D. O. Anderson, "Wireless sensor network localization techniques," Computer Networks, vol. 51, no. 10, pp. 2529-2553, 2007.

[21] B. Friedlander, "A passive localization algorithm and its accuracy analysis," IEEE Journal of Oceanic Engineering, vol. 12, no. 1, pp. 234-245, 1987.

[22] H. Schau and A. Robinson, "Passive source localization employing intersecting spherical surfaces from time-ofarrival differences," IEEE Transactions on Acoustics, Speech, and Signal Processing, vol. 35, no. 8, pp. 1223-1225, 1987.

[23] J. Smith and J. Abel, "Closed-form least-squares source location estimation from range-difference measurements," IEEE Transactions on Acoustics, Speech, and Signal Processing, vol. 35, no. 12, pp. 1661-1669, 1987.

[24] J. S. Abel and J. Smith, "The spherical interpolation method for closed-form passive source localization using range difference measurements," in ICASSP '87. IEEE International Conference on Acoustics, Speech, and Signal Processing, pp. 471-474, Dallas, TX, USA, April 1987.

[25] J. S. Abel, "A divide and conquer approach to least-squares estimation," IEEE Transactions on Aerospace and Electronic Systems, vol. 26, no. 2, pp. 423-427, 1990.

[26] W. H. Foy, "Position-location solutions by Taylor-series estimation," IEEE Transactions on Aerospace and Electronic Systems, vol. AES-12, no. 2, pp. 187-194, 1976.

[27] B. da Silva, A. Braeken, K. Steenhaut, and A. Touhafi, "Design considerations when accelerating an FPGA-based digital microphone array for sound-source localization," Journal of Sensors, vol. 2017, Article ID 6782176, 20 pages, 2017.

[28] I. Guvenc, C. C. Chong, and F. Watanabe, "Analysis of a linear least-squares localization technique in LOS and NLOS environments," in 2007 IEEE 65th Vehicular Technology Conference VTC2007-Spring, pp. 1886-1890, Dublin, Ireland, April 2007.

[29] N. Patwari, A. O. Hero, M. Perkins, N. S. Correal, and R. J. O'Dea, "Relative location estimation in wireless sensor networks," IEEE Transactions on Signal Processing, vol. 51, no. 8, pp. 2137-2148, 2003.

[30] Z. Duan and Q. Zhou, "CRLB-weighted intersection method for target localization using AOA measurements," in 2015 IEEE International Conference on Computational Intelligence and Virtual Environments for Measurement Systems and Applications (CIVEMSA), pp. 1-6, Shenzhen, China, June 2015. 


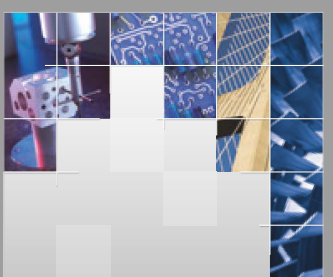

\section{Enfincering}
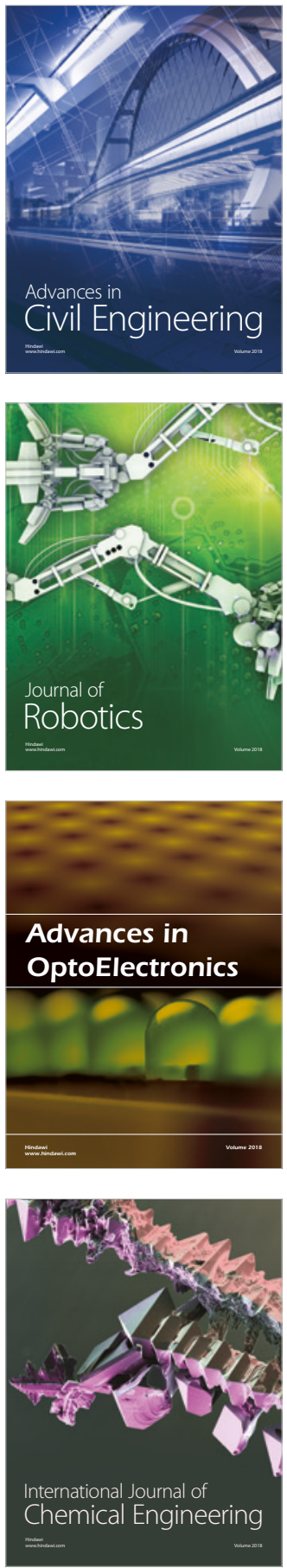

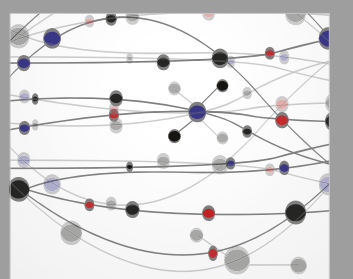

\section{Rotating \\ Machinery}

The Scientific World Journal

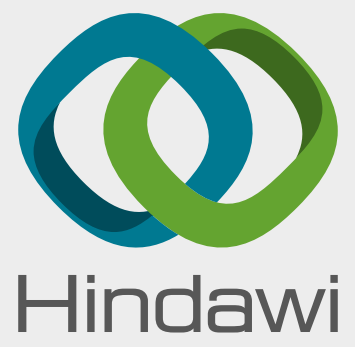

Submit your manuscripts at

www.hindawi.com
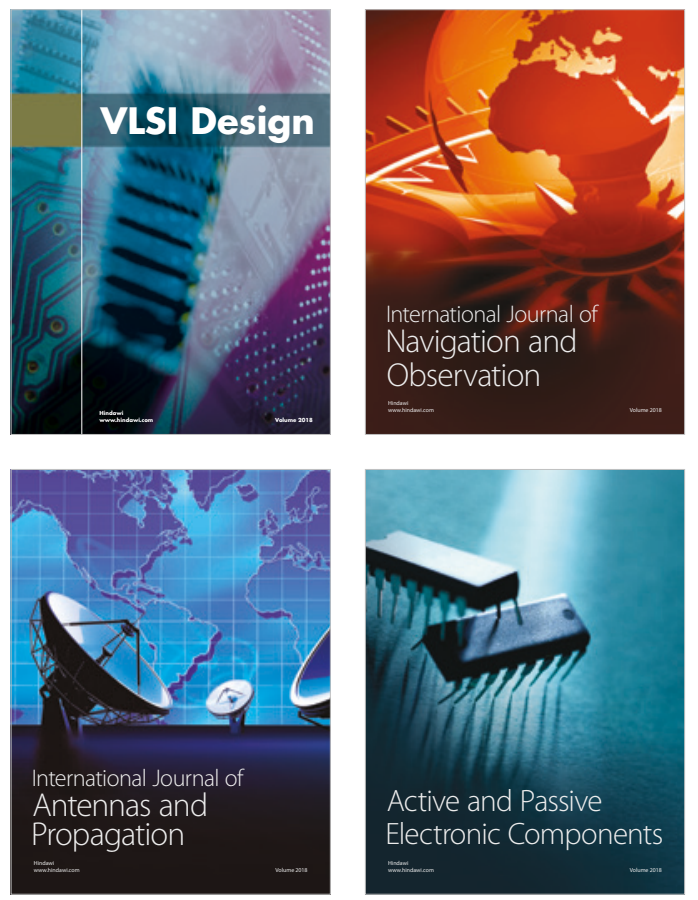
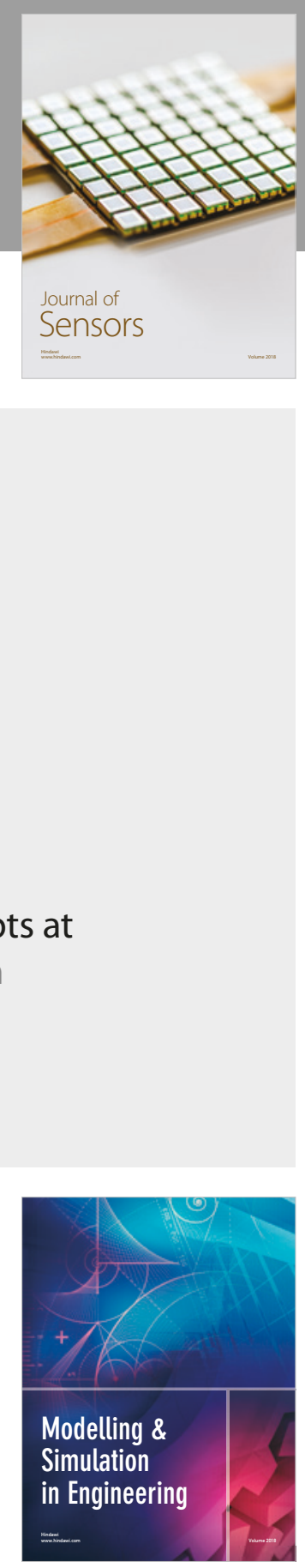

\section{Advances \\ Multimedia}
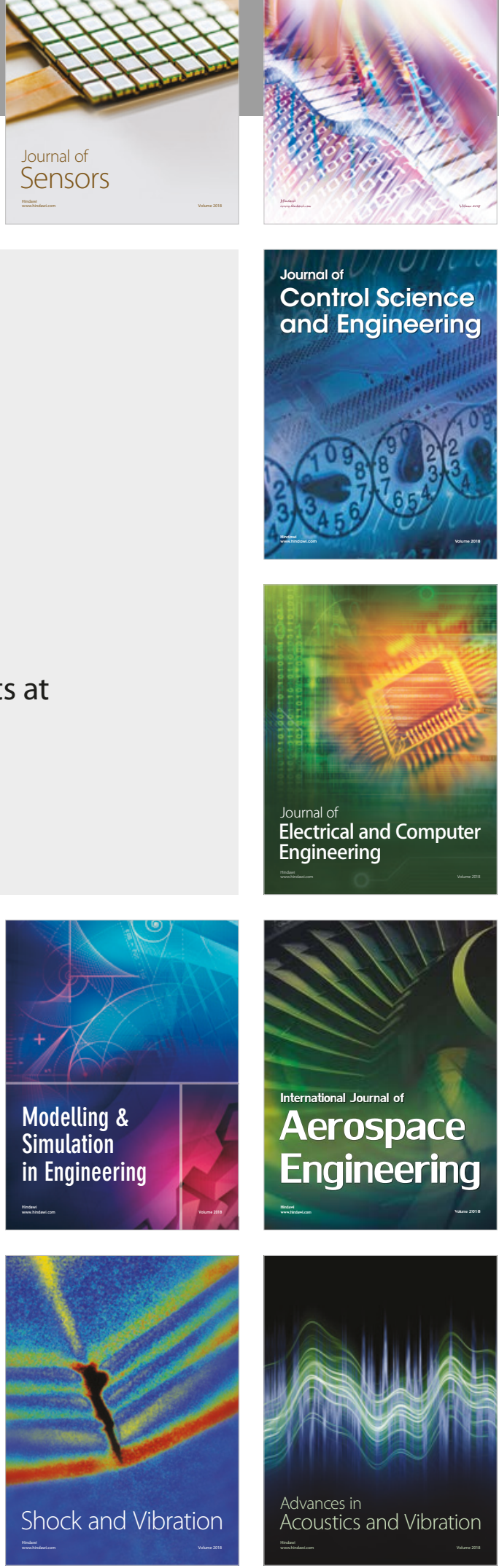